practice was inferior to 5 years in $59.3 \%$, between 5 and 10 years in $18.5 \%$, and superior to 10 years in $22.2 \%$ of cases. Sixty-three percent of rheumatologists reported that their activity decreased during covid-19 pandemic. NSAIDs prescription was avoided as much as possible in $40.7 \%$ of cases.

The participants indicated NSAIDs less frequently in $33.3 \%$ of cases, and as much as before the pandemic in one-quarter of cases.

Rheumatologists believed that NSAIDs worsen the respiratory symptoms (67\%), delay recovery (55\%), and increase mortality (48\%), hospitalization in intensive care (44\%), and infectious complications (33\%).

The participants suggested that the most incriminated NSAIDs were: Ibuprofen (7.4\%), indomethacin $(7.4 \%)$, celecoxib $(7.4 \%)$, and diclofenac $(3.4 \%)$. The majority of rheumatologists $(74 \%)$ believed that all NSAIDs had a similar risk. For patients with osteoarthritis, rheumatologists replaced NSAIDs with paracetamol and corticoids in $78 \%$ and $11 \%$ of cases, respectively. If mandatory, reducing NSAIDs doses or duration was an option in $22 \%$ and $74 \%$ of cases.

For patients with $\mathrm{SpA}$, half of rheumatologists did not change the treatment. However, the participants limited the use of NSAIDs or discontinued the treatment in patients with comorbidities. More than $60 \%$ of rheumatologists didn't know the effect of NSAIDs in the post-covid-19 syndrome.

Conclusion: Covid-19 pandemic has affected rheumatologists' practice. Rheumatic disease management during this pandemic may be challenging. More evidence is mandatory to standardize treatment prescription, especially with NSAIDs.

Disclosure of Interests: None declared

DOI: 10.1136/annrheumdis-2021-eular.3221

\section{AB0694 COVID-19 İNFECTION IN PATIENTS WITH RHEUMATIC DISEASES: TWO CENTERS EXPERIENCE}

\section{A. Sahahin ${ }^{1}$, M. A. Gedikli ${ }^{2}$, M. E. Derin ${ }^{3}$, B. Karakaş ${ }^{1}$, B. Karataş ${ }^{1}$, N. Çabuk} Çelik', İ. Yalçın' ${ }^{1}$ Sivas Cumhuriyet University, Internal Medicine Rheumatology, Sivas, Turkey; ${ }^{2}$ Sivas Cumhuriyet University, Internal Medicine, Sivas, Turkey; ${ }^{3}$ Sivas Numune Hospital, Internal Medicine - Rheumatology, Sivas, Turkey

Background: Coronavirus disease 2019 (COVID-19) is a new viral infection characterized by dry cough, fever, shortness of breath, fatigue and lymphopenia. It may be complicated by interstitial pneumonia leading to severe acute respiratory distress syndrome(1). The COVID-19 outbreak has increased concerns in those with rheumatological diseases(2).

Objectives: To present the clinical course of COVID-19 infection in patients with rheumatological disease and comorbid conditions of patients and drug use. Methods: 70 COVID-19 patients with autoimmune and autoinflammatory rheumatic diseases over the age of 18 who were followed up in the internal diseases-rheumatology department of Sivas Cumhuriyet University Hospital and Sivas Numune Hospital between April 2020 and January 2021 were included in this study. An application was made to the ethics committee of Sivas Cumhuriyet University Hospital and approval was obtained. Demographic data, comorbidities, smoking, treatments for their diseases, symptoms and hospitalization status of the patients were evaluated.

Results: The median age of the women patients was 48 years (min: 20-max: 73), 41 years in men (min: 21-max: 78$) .15$ patients (21.4\%) were male, 55 patients $(78.6 \%)$ were female. The most common rheumatic disease was rheumatoid arthritis (RA, 21, 30\%) followed by ankylosing spondylitis $(19,27.1 \%)$, familial Mediterranean fever (13, 18.6\%), Behçet's disease (12, 17.1\%). 22 (31.4\%) patients hydroxychloroquine sulfate, $14(20 \%)$ leflunomide, $7(10 \%)$ patients methotrexate, 6 (8.6\%) patients sulfasalazine, 10 (14.3\%) patients azathioprine/ mycophenolate mofetil, 22 (31.4\%) patients were using colchicine. As a biological medicine; $20(28.6 \%)$ patients were using infliximab, $5(7.1 \%)$ patients were using adalimumab, $6(8.6 \%)$ patients were using rituximab, and $4(5.7 \%)$ patients were using tocilizumab. Other are summarized in Table 1. The most common comorbidities were hypertension (25.7\%) and diabetes mellitus (DM 17.1\%). While 56 patients $(80 \%)$ had symptoms of COVID-19, 14 patients $(20 \%)$ were asymptomatic. The most common symptoms was fever $(32,45.7 \%)$ followed by cough $(29,41.4 \%)$, dyspnea $(28,40 \%)$, myalgia $(25,35.7 \%)$, loss of taste and smell $(18,25.7 \%) .21$ pneumonia cases were diagnosed. $22(31.4 \%)$ patients were hospitalized. Mortality rate was $2 / 70,2.9 \%$. Two RA patients who just treated with rituximab and tocilizumab were died. 10 cases were using $>10 \mathrm{mg} /$ day prednisolone, 2 died, 8 cases were hospitalized and had severe disease. They had hypertension and DM. Characteristics of the patients are summarized in Table 2. Conclusion: The course of COVID-19 in rheumatic diseases is not clearly. Meta-analysis has shown that the frequency of COVID-19 is increased in autoimmune diseases. However, this increase was mostly associated with the use of $>$ $10 \mathrm{mg} /$ day prednisolone. It has been stated that the use of biological drugs and Dmard causes the SARS-CoV 2 process with a milder course and therefore their use is safe (3). Similar results have been obtained in global registry registers (4). In our data, there was no increase in the mortality rate compared to the normal population. However, especially the use of rituximab may increase the rate of severe disease and mortality, so its use should be careful. There is a need for more comprehensive studies.

\section{REFERENCES:}

[1] Guan W, Ni Z, Hu Y, et al. Clinical characteristics of coronavirus disease 2019 in China. N Engl J Med 2020

[2] N. Chen, M. Zhou, X. Dong,et al., Epidemiological and clinical characteristics of 99 cases of 2019 novel coronavirus pneumonia in Wuhan, China: a descriptive study, Lancet 395 (10223) (2020) 507`513

[3] Akiyama S,Hamdeh S, Micic D, et al, Prevalence and clinical outcomes of COVID-19 in patients with autoimmune diseases: a systematic review and meta-analysis,Ann Rheum Dis 2020 Oct 13;annrheumdis-2020-218946.

[4] Gianfrancesco M, Hyrich KL, Al-Adely S,et al, Characteristics associated with hospitalisation for COVID-19 in people with rheumatic disease: data from the COVID-19 Global Rheumatology Alliance physician-reported registry. Ann Rheum Dis. 2020 Jul;79(7):859-866.

Disclosure of Interests: None declared

DOI: 10.1136/annrheumdis-2021-eular.3289

\section{AB0695 19 PATTERN OF COVID-19 IN PATIENTS WITH RHEUMATIC DISEASES UNDERGOING BIOLOGICAL THERAPY: A COHORT EXPERIENCE}

C. Ancuta ${ }^{1,2}$, C. Pomirleanu ${ }^{1,2}$, G. Strugariu ${ }^{2}$, L. Petrariu ${ }^{2}$, E. Ancuta ${ }^{3}$,

C. $\operatorname{Bran}^{4,5}$, R. Chirieac ${ }^{6}$, C. Mihailov ${ }^{7,8}$. ${ }^{1}$ University of Medicine and Pharmacy "GRIGORE T. POPA"IASI, Rheumatology, IASI, Romania; ${ }^{2}$ Clinical Rehabilitation Hospital, Rheumatology, IASI, Romania; " "Elena Doamna"Clinical Hospital, Research, IASI, Romania; ${ }^{4}$ Emergency Hospital "Sf loan", Rheumatology, IASI, Romania; ${ }^{5}$ University Stefan Cel Mare Suceava, Semiology and Immunology, Suceava, Romania; ${ }^{6}$ Sanocare Medical and Research Center, Rheumatology, IASI, Romania; ${ }^{7}$ Clinical CF Hospital of Constanta, Rheumatology, Constanta, Romania; ${ }^{8}$ Faculty of Medicine, "Ovidius" University Constanta, Internal Medicine and Rheumatology, Constanta, Romania

Background: Despite emerging vaccines, the world is in the midst of a coronavirus disease 2019 (COVID-19) pandemic. Outcomes of SARS-CoV2 infection remain a major concern in patients with rheumatic and musculoskeletal diseases, especially for those with uncontrolled disease.

Objectives: We aimed to investigate trends and outcomes of COVID-19 occurring in patients with chronic inflammatory rheumatic conditions treated with biologics and targeted synthetic disease modifying antirheumatic drugs (bDMARDs, tsDMARDs).

Methods: We included all confirmed cases of COVID-19 regardless of severity in patients with rheumatoid arthritis (RA), ankylosing spondylitis (AS) and psoriatic arthritis (PSA) undergoing bDMARDs or tsDMARDs treatment registered in our local COVID-19 reporting database. We collected relevant information about comorbidities, rheumatologic-related clinical activity (RAPID5, SDAI, BASDAI DAPSA), type of DMARD and glucocorticoid use, as well as COVID-19 related data as severity (ranging from asymptomatic to life-threatening forms), medication, hospitalization, intensive care unit admission, invasive mechanical ventilation and death.

We did a subgroup analysis among patients with a specific rheumatologic diagnosis, among different class of medication, patients who were or not hospitalized with COVID-19 looking how age, comorbidities, type of rheumatic condition and treatments impact COVID outcomes.

Results: 40 COVID-19 cases (positive PCR for SARS-CoV2) (4.67\%) were identified during the 6-month study period among 855 patients registered in our database of patients under biologic treatment, including 20 RA, 18 SpA, 2 PsA patients. The majority were in either low disease activity or remission, only two patients had active uncontrolled disease at the onset of coronavirus infection. 16 cases $(40 \%)$ were asymptomatic and were tested RT-PCR-positive during routine follow-ups for their disease, 13 cases (32.5\%) had mild and 8 cases (20\%) moderate illness; severe pneumonia and critical disease with acute respiratory distress syndrome were reported in only 3 cases, 2 recovered after; the only patients who died was 69 years old, had cardiac disease, hypertension and diabetes, had undertaken regular rituximab perfusion one month before coronavirus infection and developed pulmonary embolism followed by septic shock. Extreme fatigue was the dominant COVID-19 associated symptom apart from the classical ones including fever, cough, shortness of breath, sore throat, nasal congestion, headache, anosmia and ageusia myalgias and anorexia.

No specific pattern for patients requiring intensive care unit admission.

Conclusion: The COVID-16 infection rate in patients with inflammatory rheumatic disorders receiving biologics and tsDMARDs is pretty low; although immu nosuppressed, these patients seem not to be at risk for severe COVID-19 illness and outcomes. These findings might reflect a potential protective role of certain biologics and/or JAK inhibitors for development and severity of COVID-19 in patients. 
REFERENCES:

[1] WHO, COVID-19 clinical management, Living Guidance, 25 Jan 2021 Disclosure of Interests: CODRINA ANCUTA Speakers bureau: Abbvie, Pfizer, Lilly, Novartis, Sandoz, Consultant of: Abbvie, Pfizer, Lilly, Novartis, Sandoz, Cristina Pomirleanu Speakers bureau: Abbvie, Pfizer, Lilly, Novartis, Sandoz, Georgiana Strugariu Speakers bureau: Abbvie, Pfizer, Lilly, Novartis, Sandoz, Luiza Petrariu Speakers bureau: Abbvie, Pfizer, Lilly, Novartis, Sandoz, Eugen Ancuta: None declared, Codruta Bran Speakers bureau: Abbvie, Pfizer, Lilly, Novartis, Sandoz, Rodica Chirieac Speakers bureau: Abbvie, Pfizer, Lilly, Novartis, Sandoz, Claudia Mihailov Speakers bureau: Abbvie, Pfizer, Lilly, Novartis, Sandoz, Consultant of: Abbvie, Pfizer, Lilly, Novartis, Sandoz

DOI: 10.1136/annrheumdis-2021-eular.3467

\section{AB0696 ANTI-PHOSPHOLIPID AUTOANTIBODIES IN COVID-19 PATIENTS}

M. Jordhani', D. Ruci ${ }^{1}$, V. Ruci'. ${ }^{1}$ UHC Mother Teresa, Tirana, Albania, Internal Medicine, Tirana, Albania; ${ }^{2}$ University Trauma Hospital Tirana, Orthopaedics, Tirana, Albania

Background: Because of the inflammation boosting cytokines, Coronavirus disease 2019 (COVID-19) has demonstrated thrombotic consequences that have increased its morbidity and mortality. There is evidence that mechanisms that contribute in thrombosis in COVID-19 patients are similar to those in anti-phospholipid syndrome (aPS). In fact, there is a possibility that anti-phospholipid autoantibodies (aPLs) might impulse thrombosis in patients with COVID-19, as literature suggests ${ }^{2}$.

Objectives: The aim of our study was to evaluate the anti-phospholipid autoantibody titre in patients with COVID-19 during and after the infection.

Methods: This is an observational study which included 71 patients with a recent COVID-19 up to 4 weeks after. Every patient was completed with aPL titre about IgG and IgM anti-cardiolipine (ACA) and lupus anticoagulant (LAC) autoantibodies. According to titre results, the patients were divided into groups in order to better show the immunologic results.

Results: After gathering and analysing the data, it was estimated that 21 patients (29.6\%) were positive for at least one type of aPL antibody: 12 patients were found positive for lupus anticoagulant autoantibodies (57.1\%), 6 patients were double positive for LAC and ACA $(28.6 \%)$, and 3 patients were positive for anti-cardiolipin antibodies (14.3\%). Seven patients were IgM positive for any aPL (33.3\%), 6 patients were found to have positive $\lg$ M and $\operatorname{lgG}(28.6 \%)$ and 8 patients had only IgG antibodies (38.1\%).

Conclusion: From this study it was observed that a significant proportion of patients with recent COVID-19 infection had positive anti-phospholipid antibodies, compared to the general population prevalence. This suggests that the impact of aPLs in COVID-19 might be of great importance. It should be carefully evaluated in order to better understand the mechanisms of thrombotic complications.

REFERENCES:

[1] Wise Jacqui. Covid-19 and thrombosis: what do we know about the risks and treatment? BMJ 2020; 369:m2058

[2] Zuo, Yu, et al. "Prothrombotic autoantibodies in serum from patients hospitalized with COVID-19." Science translational medicine 12.570 (2020).

Disclosure of Interests: None declared

DOI: 10.1136/annrheumdis-2021-eular.3521

\section{AB0697 ANTI-SARS-COV-2 ANTIBODIES AND AUTOANTIBODIES IN COVID-19 PATIENTS SURVIVED AFTER ICU ADMISSION, 6 MONTHS LATER}

K. Bitzogli ${ }^{1}$, E. Magira ${ }^{2}$, L. Chatzis' ${ }^{1}$, E. Jahaj ${ }^{2}$, H. Alexopoulos ${ }^{1}$, M. Dalakas ${ }^{1}$, A. Kotanidou', A. Tzioufas ${ }^{1}$, P. Vlachoyiannopoulos ${ }^{1} .^{1}$ School of Medicine, National and Kapodistrian University of Athens, Pathophysiology, Athens, Greece; ${ }^{2}$ School of Medicine, National and Kapodistrian University of Athens, 1st Department of Intensive Care Medicine, Athens, Greece

Background: $\mathrm{We}^{1}$ and others ${ }^{2}$ have previously shown that ICU admitted patients with COVID-19 developed high titers of anti-SARS-CoV-2 antibodies, but also autoantibodies, some of which are pathogenic. We re-evaluated 8 patients of those survived after admission to the ICU of Evangelismos Hospital of Athens $-1^{\text {st }}$ Department of Internal Medicine, Medical School, NKUA ${ }^{3} 6$ months later. We did not know whether these autoantibodies still exist, are associated with COVID19 or with ARDS as described after septic shock ${ }^{4}$.

Objectives: To investigate the presence and titers of anti-SARS-CoV-2 antibodies and autoantibodies in patients survived after COVID-19 ICU stay, in the ICU and 6 months later.

Methods: Case series to evaluate titers of anti-SARS-CoV-2 antibodies, specificities of autoantibodies as well as clinical features in ICU admitted COVID-19 patients, initially and 6 months after their discharge. Evaluation of current clinical status included evaluation of lung, heart, kidney, central and peripheral nervous system and mental status using standardized methods. Methods for detection of anti-SARS-CoV-2 antibodies and autoantibodies were described in our previous report ${ }^{1}$

Results: We had initially evaluated ${ }^{1} 29$ ICU admitted COVID-19 patients' files and sera, of which 4 had been already died during serum evaluation. Six more patients died thereafter. Out of 19 having been discharged, 8 were willing to be re-evaluated. On second evaluation 6 months later, serum anti-SARS-CoV-2 antibodies were highly positive, although at lower titers compared to the titers at disease onset (median [range]) 8.705 (range: 7.95-9.56) vs 6.640 (range: 6.29 6.76), $p=0.0002$, Mann-Whitney test. Initially 3 out of 8 patients expressed antinuclear antibodies (ANA) at titers $1 / 160,1 / 320$ and $1 / 320$ with a fine speckled pattern with the second patient also expressing at a titer of $1 / 160$, antimitochondrial (AMA) antibodies. Six months later the same patients and not anyone else expressed ANA of the same pattern at titers $1 / 640,1 / 160$ and $1 / 160$ respectively. Two patients with $1 / 20 \mathrm{p}$-ANCA and $1 / 640 \mathrm{c}$-ANCA initially, lost their respective autoantibodies after 6 months. One patient initially negative for IgM anti-ß2GP became positive at low titer and an initially positive became negative. One patient initially positive for anti-Ro60 antibody continued to be positive 6 months later. One patient initially negative developed anti-Tg antibodies and 3 patients initially positive for anti-TPO antibodies remained positive 6 months later.

Conclusion: Patients with COVID-19 survived after ICU admission still retain high titers of anti-SARS-CoV-2 antibodies but significantly lower that at disease onset, but they tend to lose autoantibodies with pathogenic potential.

\section{REFERENCES:}

[1] Vlachoyiannopoulos P et al, Ann Rheum Dis 2020,

[2] 2. Wang $E Y$ et al, medRxiv preprint doi: https://doi. org/10.1101/2020.12.10.20247205

[3] National and Kapodistrian University of Athens, Athens, Greece

[4] Burbelo et al. Journal of Translational Medicine 2010

Disclosure of Interests: None declared

DOI: 10.1136/annrheumdis-2021-eular.3554

\section{AB0698 CORONAVIRUS 19 DISEASE VACCINE: PERCEPTIONS AND INTENTIONS OF TUNISIAN PATIENTS WITH RHEUMATOID ARTHRITIS}

K. Ben Abdelghani ${ }^{1}$, H. Boussaaa ${ }^{1}$, S. Miladi ${ }^{1}$, M. Sellami ${ }^{1}$, L. Souabni ${ }^{1}$, K. Ouenniche ${ }^{1}$, S. Kassab ${ }^{1}$, S. Chekili', A. Fazaa ${ }^{1}$, A. Laatar ${ }^{1} .{ }^{1}$ Mongi Slim University Hospital, Rheumatology, La Marsa, Tunisia

Background: Coronavirus disease 2019 (covid-19) has now spread to millions of persons worldwide to become a global pandemic. Covid-19 is asymptomatic for some individuals and for others it can cause symptoms ranging from flu-like to acute respiratory distress syndrome, pneumonia and death. Several vaccine candidates are now available, and patients with chronic inflammatory disease such as rheumatoid arthritis (RA) are encouraged to get vaccinated.

Objectives: The aim of this study was to determine perceptions of RA patients about the covid-19 vaccine.

Methods: We conducted a cross-sectional study including Tunisian patients with RA (ACR/EULAR 2010). Demographic and disease parameters were collected: age, gender, educational status, disease duration, erythrocyte sedimentation rate (ESR), disease activity score (DAS28), and treatments being used. All patients responded to a questionnaire on their perceptions and concerns about the covid-19 vaccine, and whether they intended to get vaccinated or not. A $p$ value inferior to 0.05 was considered significant.

Results: We included 54 patients ( 45 women and nine men) with a mean age of $55 \pm 11$ years old [23-69]. Thirty-one percent of patients were illiterate. The mean disease duration was $9.9 \pm 5.9$ years $[0-20]$. The mean DAS28 ESR was $4.68 \pm 1.35$ [1.50-7.16].

NSAIDs were used in $13 \%$ of patients, corticosteroids in $63 \%$ of patients at a mean daily dose of $8 \mathrm{mg}$ [2.5-20] of prednisone equivalent, methotrexate in $63 \%$ of patients, sulphasalazine in $13 \%$ of patients, leflunomide in $3.7 \%$, and biologics in $22.2 \%$ of patients.

None of these patients had contracted the covid-19. Seventeen percent of patients had been in close contact with someone positive for the disease. All the patients reported that they respected the preventive measures. Fifteen percent of patients had stopped their treatment because they were afraid of the covid19: Methotrexate $(n=4)$, sulphasalazine $(n=1)$, tocilizumab $(n=2)$, and rituximab $(n=1)$.

More than half of patients $(67.7 \%)$ reported that they didn't want to get vaccinated against covid-19. The reasons given by these patients were: presumed adverse events $(100 \%)$, religious beliefs $(86.7 \%)$. presumed inefficiency $(83.3 \%)$, no recommendation from their doctor $(80 \%)$, fear that the vaccine would interact with their treatment $(76.7 \%)$, lack of trust in the pharmaceutical laboratories $(76.7 \%)$, fear from vaccines in general $(53.3 \%)$, fear that the vaccine would make RA worse $(50 \%)$, and presumed overprice of the vaccine $(30 \%)$. 